terms of increased crop yields, were obtained (chiefly on horticultural crops) but they were erratic.

The development of current understanding of the mode of action of different soil conditioners was described by Professor D. J. Greenland (University of Reading). The characteristics of structures to be conditioned were outlined; for example, pores which are large enough for bacteria to enter but which will also hold "available" water must be stabilized. Dr. F. De Bisschop (University of Ghent) described his work on the mechanism of the binding process between soil particles with emulsions based on products of the petroleum industry.

One important aspect stressed at the conference was the need for a multidisciplinary approach if problems concerned with the development of good stable structures were to be solved. The help that organic chemists could give was illustrated by Professor N. Schamp's (University of Ghent) contribution on the physico-chemical interaction of polyacrylamide and clay particles. Emphasis has now shifted away from such conditioners as hydrolysed polyacrylonitrile to polyacrylamide and polyvinyl alcohol. Polymers need to be sufficiently small to enter the pores of the soil which requires stabilization.

The need for producing conditioners which are not harmful to the environment was stressed, and Professor Schamp described the work of his department on checking potential conditioners for carcinogenicity.

If work on conditioners is to be developed it is necessary to understand what happens when soil structures break down in field conditions. Professor G. Bolt (State Agricultural University, Wageningen) gave an outline of theoretical aspects of swelling and other destructive forces on soil aggregates and was followed by Dr F. Koenigs of the same university, who talked about practical aspects of structure deterioration.

The structure of the soil determines water infiltrability and storage. If the structure is unstable, particularly at the surface, infiltrability is reduced, runoff is increased and erosion either commences or is increased. Dr G. Vachaud (University of Grenoble) described sophisticated methods for continuous assessment of soil moisture in the field and was followed by Professor W. $\mathbf{H}$. Gardner (Washington State University) on erosion control and moisture conservation in dryland farming. Professor D. A. Farrell (Iowa State University) discussed the effect of soil infiltration and runoff in crusted and uncrusted soils. An advance would be made if conditioners could be used in small amounts for treating the "skin" on soils tending to crust so that instead of runoff water movement into them could occur but would still allow seedling emergence.

Mathematical models were discussed by several speakers, who included Professor W. R. Gardner (University of Wisconsin) on prediction of moisture transmission in conditioned soils; Professor G. Van Steenkiste (University of Ghent) on the study of moisture flow by hybrid computer; and Professor J. Gani (University of Sheffield) on mathematical models in agriculture.

At the final session an attempt was made by Professor W. R. Gardner to draw together the wide range of topics discussed. One of the reasons for the loss of interest in soil conditioners in the $1950 \mathrm{~s}$ was their high cost. Being relatively complex molecules, the cost of conditioners of the type at present under investigation must remain high. Evidence was produced during the meeting that it may be possible to stabilize soils with much smaller amounts of material. At present, the current approach in the field is to concentrate on relatively small scale problems-for example, stabilization of road banks during the construction of new motorways and the control of sand dunes in semi-urban areas where land values are high. By concentrating work in this much more limited field, it is possible to consider products which are much more costly than are normally acceptable in agriculture.

\section{PLATE TECTONICS}

\section{lithospheric Tearing}

from our Geomagnetism Correspondent

As the spreading north Pacific approaches the Aleutian Island arc it is presented with a trench which is generally convex towards the south. In greater detail, the Aleutian trench appears as three almost linear segments with two fairly abrupt changes in direction. Thus at about $180^{\circ}$ longitude, just south of the Bowers Ridge, a $40^{\circ}$ change in the trend of the trench axis separates the Western and Central Aleutians; and at about $170^{\circ}$ longitude a rather smaller change of about $20^{\circ}$ marks the boundary between the Central and Eastern Aleutians. In view of these trends, an obvious question to ask concerns the nature of the tectonic processes occurring at the two discontinuities. In particular, how does the spreading lithosphere react as it reaches, and descends into, a trench which presents an angular, as opposed to a linear, front.

According to Abe (Earth Planet. Sci. Lett., 14, 428; 1972), seismological evidence suggests that at the $40^{\circ}$ discontinuity, at least, the lithosphere bends and tears. The basic data to support this conclusion come from an analysis of the travel times from the Longshot underground nuclear explo-

\title{
Action of Calcium in Muscle
}

IN next Wednesday's Nature New Biology (June 14), Ashley and Moisescu present a model for the action of calcium ions in controlling tension in muscle. The development of tension at relatively large calcium concentrations and over short time intervals depends on the square of the calcium concentration, and it is inferred that the process involves two distinct calcium sites. Earlier evidence on the rate of development of tension also indicated that two separate slow processes contribute to it.

Ashley and Moisescu now examine the fit of available data to simple equations based on the following models: simultaneous binding of two calcium ions in a slow reaction, followed by a further slow rate process; separate binding of the two calcium ions, that of one being contingent on prior binding of the other; separate binding of two ions at different sites that affect the same functional unit of the myofibril.

The first scheme provides a relatively poor fit to biochemical and binding data. The third model is treated in detail, and gives a satisfactory fit of the transient kinetics and also the steadystate characteristics of the ATPase.
Under narrowly defined conditions the ATPase shows an increase over a range of calcium concentration which is tenfold greater than normal, suggesting an uncoupling of the two binding processes, and corresponding predictions from the model are borne out. The quantitative relation between free calcium concentration and the luminescent response of aequorin, which is used to assay it, is also considered. The treatment indicates that the isometric tension can under all conditions be determined directly by the concen. tration of free calcium ions.

In the same issue of Nature New Biology, Endo describes the activation of skinned muscle fibres by low concentrations of calcium ions. He has found that the development of isometric tension under these conditions can be made to increase when the fibre is stretched. Three reasonable explanations for this finding are offered: increased binding of calcium to troponin when the length is greater; stronger interaction between thick and thin filaments in the stretched fibre, because they will then be closer together ; or some change in the nature of the actin-myosin interaction when the filaments are under tension. 